\title{
Preventive Effect of Shenduning Prescription Combined with Hydration Therapy on Contrast Induced Nephronpathy after Elective Percutaneous Coronary Intervention
}

\author{
Jiangang Jiang ${ }^{1 *}$, Xianqing $\mathrm{Hu}^{2}$, Ningning $\mathrm{Ji}^{3}$, Chengyuan Yan ${ }^{1}$, Jingliang Lan ${ }^{1}$, Xiaoma Du ${ }^{1}$ \\ ${ }^{1}$ Jinhua Hospital of Traditional Chinese Medicine Affiliated to Zhejiang Chinese Medical University, Jinhua 321017, Zhejiang, China \\ ${ }^{2}$ Jinhua Municipal Central Hospital, Jinhua 321000, Zhejiang, China \\ ${ }^{3}$ Yiwu Central Hospital, Jinhua 322099, Zhejiang, China \\ Email: jiangjiangang2020@163.com
}

\begin{abstract}
Objective - To explore the preventive effect of Shenduning prescription combined with hydration therapy on contrast induced nephronpathy (CIN) after elective percutaneous coronary intervention. Methods - 159 patients undergoing selective coronary intervention were divided into simple hydration group, intensive statin group and Shenduning prescription group randomly, with 53 cases in each group. The simple hydration group received conventional drugs for coronary heart disease and preoperative routine hydration therapy. The intensive statin group received intensive treatment with atorvastatin calcium tablets on the basis of the simple hydration group, and the Shenduning prescription group received Shenduning prescription treatment on the basis of the hydration group. Serum creatinine (Scr), malondialdehyde (MDA), superoxide dismutase (SOD) and glomerular filtration rate (eGFR) were detected by blood samples at $24 \mathrm{~h}$ before and after operation, as well as $72 \mathrm{~h}$ after operation respectively. The primary endpoint event was the occurrence of CI-AKI. Results - The Scr level of the intensive statin group and the Shenduning prescription group was significantly lower than that of the simple hydration group, while the eGFR level was significantly higher than that of the simple hydration group, which is of statistical significance $(\mathrm{P}<0.05)$. The incidence of CI-AKI was $15.1 \%$ in simple hydration group, $5.7 \%$ in intensive statin group and $3.8 \%$ in Shenduning prescription group respectively after operation $\left(x^{2}=5.194, \mathrm{P}=0.075\right)$, of which there was significant statistical difference between simple hydration group and Shenduning prescription group $\left(x^{2}=3.975, \mathrm{P}=0.046\right)$. After $72 \mathrm{~h}$ of the operation, MDA level in Shenduning prescription group was significantly lower than that in simple hydration group and intensive statin group, while its SOD level was significantly higher than that in simple hydration group and intensive statin group, which is of statistical significance $(\mathrm{P}<0.05)$. The proportion of abnormal liver function in intensive statin group and the Shenduning prescription group was $13.1 \%$ and $3.8 \%$, respectively, and the difference was statistically significant $\left(x^{2}=4.867, \mathrm{P}=0.027\right)$. Conclusion - The combination treatment of Shenduning prescription with hydration therapy may protect the patient's renal function after elective coronary interventional therapy, while reducing the incidence of CIN and the oxidative stress reaction caused by contrast agent, thus effectively reducing adverse reactions.

Keywords: percutaneous coronary intervention, hydration therapy, Shenduning prescription, contrast induced nephronpathy, glomerular filtration rate
\end{abstract}

\section{Introduction}

In recent years, with the aggravation of population aging in the whole society, percutaneous coronary intervention has widely developed in primary hospitals, which has brought great benefits for the cardiovascular health of the public. However, the side effects caused by contrast agent during percutaneous coronary intervention cannot be ignored, especially the occurrence of contrast-induced nephropathy (CIN), which significantly affects the prognosis of patients ${ }^{[1]}$. Studies have confirmed ${ }^{[1-3]}$ that the incidence of CIN in the general population is $5.8 \%$, but it is higher than $50 \%$ in some high-risk groups, which must be taken seriously by relevant personnel. However, the exact mechanism of CIN occurrence is still unknown, and it is generally accepted that it may have certain relationship with the renal medullary ischemia and hypoxia, renal damage caused by oxygen free radical, toxic effect of contrast agent and inflammatory reaction ${ }^{[4-6]}$. According to different pathogenesis, various targeted drugs are emerging in clinical practice. Although all of them have certain clinical effects, no drugs that can completely prevent the occurrence of CIN have been found in clinical practice so far. Therefore, a combination therapy of traditional Chinese and western medicine may be a breakthrough in the prevention and treatment of CIN. Shenduning prescription, originally created by Professor Fu Xiaojun, a famous traditional Chinese medicine inheritor, 
has definite efficacy in the treatment of chronic renal failure, but there lacks relevant clinical research on the prevention and treatment of CIN. This study is a random, contrast and prospective clinical study, aiming to evaluate the incidence of CIN applying the combination treatment of Shenduning prescription with hydration therapy after elective percutaneous coronary intervention and its clinical application value in protecting patient's renal function. The report is as follows.

\section{Research subjects and methods}

\subsection{Research subjects}

This study is designed in a prospective, random and contrast way. A total of 159 patients undergoing selective coronary intervention in the Department of Cardiology in Jinhua Hospital of TCM, Yiwu Central Hospital and Jinhua Central Hospital of Zhejiang Province from January 2020 to May 2021 were selected as research subjects, including 101 males and 58 females, with an average age of (60.71 \pm 7.82$)$ years old. And they were randomly divided into three groups: simple hydration group, intensive statin group and Shenduning prescription group, with 53 cases in each group. Inclusion criteria: (1) cardiac function $\leq$ III (HYHA classification); (2) Patients with glomerular filtration rate (eGFR) $\geq 60 \mathrm{~mL} / \mathrm{min} / 1.73 \mathrm{~m} 2$ and no primary renal diseases; (3) No drugs affecting renal function have been used in the past month. Exclusion criteria: (1) patients with non-elective coronary intervention; (2) Patients with critical condition or unstable vital signs, such as cardiogenic shock, NYHA cardiac function grade IV, malignant arrhythmia, acute hypertension and other diseases; (3) Complicated with other important organ diseases, such as severe liver and kidney insufficiency, cerebrovascular accident within 12 months, uncontrolled severe diabetes, gastrointestinal bleeding, infectious diseases and tumors; (4) patients who are allergic to contrast agents, and unwilling to take Chinese herbal decoction, as well as involuntary participants. This study was approved by the Ethics Committee of Jinhua Hospital of TCM in Zhejiang Province, and the informed consent was signed all research subjects.

\subsection{Research methods}

All subjects were divided into three groups randomly, namely, simple hydration group, intensive statin group and Shenduning prescription group. In simple hydration group, intravenous infusion of $0.9 \%$ sodium chloride solution at a dose of $1.5 \mathrm{ml} / \mathrm{kg} / \mathrm{h}$ was used $6 \sim 12 \mathrm{~h}$ before coronary intervention and during the operation, and the dose was increased to $2.5 \mathrm{ml} / \mathrm{kg} / \mathrm{h}$ within $6 \mathrm{~h}$ after surgery. In intensive statin group, $40 \mathrm{mg}$ Atorvastatin calcium tablets were taken orally once a day before bed (Shandong Qilu Pharmaceutical Co., Ltd., 20mg/tablet, National Drug Approval: H20193144) 72h before and after operation respectively on the basis of treatment in the simple hydration group. In Shenduning prescription group, Shenduning granules (drug composition: astragalus membranaceus $30 \mathrm{~g}$, herba epimedii $20 \mathrm{~g}$, prepared rhubarb $15 \mathrm{~g}$, Salvia miltiorrhiza $15 \mathrm{~g}$, Chinese agarwood powder $2 \mathrm{~g}$, Peach kernel $10 \mathrm{~g}$, prepared sealwort $20 \mathrm{~g}$, radix pseudostellariae $15 \mathrm{~g}$, and cassia twig 9g; provided by TCM Granules of Jinhua Hospital of TCM) was added $72 \mathrm{~h}$ before and after operation on the basis of treatment in the simple hydration group. Each prescription was divided into two doses and shall be taken in the morning and evening with $200 \mathrm{ml}$ warm water.100ml. Iopadol injection (National Drug Approval: H20053388) was used as the contrast agent during the operation, with an iodine content of $370 \mathrm{mg} \mathrm{I} / \mathrm{ml}$.

\subsection{Data collection and testing}

The general information of the patients was recorded, mainly including gender, age, history of hypertension, history of diabetes, history of hyperlipidemia, smoking history, coronary angiography results, operative time, contrast agent dosage, etc. $5 \mathrm{ml}$ of peripheral venous blood was collected from all subjects on the morning after admission, $24 \mathrm{~h}$ and $72 \mathrm{~h}$ after operation, respectively, and CIN was calculated according to the postoperative Scr peak of patients. The diagnostic criteria for $\mathrm{CIN}^{[7]}$ : serum Scr value increased $\geq 0.5 \mathrm{mg} / \mathrm{dl}(44.2 \mu \mathrm{mol} / \mathrm{L})$ in contrast with baseline absolute value or relative value increased $\geq 25 \%$ within $72 \mathrm{~h}$ after exposure to iodine contrast agent, excluding renal function impairment caused by other reasons. Main observation indexes: (1) Kidney injury markers include serum creatinine (Scr) and glomerular filtration rate (eGFR). eGFR was calculated using a simplified diet adjustment study formula for renal disease. The estimated eGFR (ml/ $\mathrm{min} / 1.73 \mathrm{~m} 2)=175 \times \mathrm{Scr}(\mathrm{mg} / \mathrm{dl})-1.154 \times$ age $-0.203 \times(0.79$ female $) ;(2)$ Markers of oxidative stress, include malondialdehyde (MDA) and superoxide dismutase (SOD); (3) The incidence of renal dialysis and abnormal liver function after operation was recorded in each group. Abnormal liver function refers to normal liver function the next day after admission while abnormal liver function $72 \mathrm{~h}$ after operation; Or liver function deteriorates $72 \mathrm{~h}$ after operation compared with the first time liver deterioration after admission. 


\subsection{Statistical methods}

SPSS 23.0 software was used for data statistics. The measurement data of normal distribution were expressed as $( \pm \mathrm{s})$, and analysis of variance was used for comparison among the three groups. Counting data were represented as example (\%). Chi-square test was used for comparison between groups. If any theoretical number $\mathrm{T}<5$, Fisher exact test was used. Repeated measurement analysis of variance was used to compare the data of different groups at different time points, and the $\mathrm{q}$ test was used for pairwise comparison. Paired $\mathrm{t}$ test was used for comparison before and after treatment. $\mathrm{P}<0.05$ was considered statistically significant.

\section{Results}

\subsection{Comparison of baseline data of the three groups}

There were no significant differences in age, gender and body mass index (BMI) among the three groups (P $>0.05)$; And there were no significant differences in cardiovascular risk factors among the three groups $(\mathrm{P}>0.05)$; Moreover, there was no statistical significance in the severity of coronary artery disease, operation time, contrast agent dosage and other aspects among the three groups $(\mathrm{P}>0.05)$. See Table 1 .

\begin{tabular}{|c|c|c|c|c|c|}
\hline Items & $\begin{array}{l}\text { Simple hydration group } \\
\qquad(n=53)\end{array}$ & $\begin{array}{l}\text { Intensive statin group } \\
\qquad(n=53)\end{array}$ & $\begin{array}{c}\text { Shenduning } \\
\text { prescription group } \\
(n=53)\end{array}$ & $\begin{array}{l}\mathrm{F} / x^{2} \\
\text { value }\end{array}$ & $\begin{array}{c}\mathrm{P} \\
\text { value }\end{array}$ \\
\hline \multicolumn{6}{|l|}{ Demographic characteristic } \\
\hline Male (\%) & $34(64.2)$ & $36(67.9)$ & $37(69.8)$ & 0.400 & 0.819 \\
\hline age (years) & $60.34 \pm 7.18$ & $59.74 \pm 8.22$ & $60.81 \pm 7.59$ & 0.246 & 0.734 \\
\hline $\operatorname{MBI}\left(\mathrm{kg} / \mathrm{m}^{2}\right)$ & $25.19 \pm 1.74$ & $24.79 \pm 1.58$ & $25.06 \pm 1.64$ & 0.317 & 0.736 \\
\hline \multicolumn{6}{|l|}{ Cardiovascular risk factors } \\
\hline Hypertension $(\%)$ & $36(67.9)$ & $33(62.3)$ & $35(66.0)$ & 0.389 & 0.823 \\
\hline Diabetes $(\%)$ & $18(34.0)$ & $14(26.4)$ & $15(28.3)$ & 0.785 & 0.675 \\
\hline Hyperlipidemia (\%) & $19(35.8)$ & $22(41.5)$ & $26(59.1)$ & 1.909 & 0.385 \\
\hline Smoking $(\%)$ & $25(47.2)$ & $34(64.3)$ & $31(58.5)$ & 3.226 & 0.199 \\
\hline \multicolumn{6}{|l|}{ Conditions of Coronary angiography } \\
\hline None vessel $(\%)$ & $4(7.5)$ & $2(3.8)$ & $4(7.5)$ & 6.716 & 0.404 \\
\hline Single vessel $(\%)$ & $29(54.7)$ & $23(43.4)$ & $20(37.7)$ & & \\
\hline Double vessel $(\%)$ & $14(26.4)$ & $24(45.3)$ & $24(45.3)$ & & \\
\hline Triple vessel $(\%)$ & $6(11.3)$ & $4(7.5)$ & $5(9.4)$ & & \\
\hline Mean operation time $(\mathrm{min})$ & $38.47 \pm 22.28$ & $40.81 \pm 25.34$ & $35.23 \pm 27.46$ & 0.943 & 0.613 \\
\hline Average amount of contrast agent $(\mathrm{ml})$ & $80.26 \pm 34.13$ & $82.54 \pm 30.44$ & $80.17 \pm 28.73$ & 0.331 & 0.764 \\
\hline
\end{tabular}

\subsection{Comparison of markers of renal injury in the three groups}

There were no statistically significant differences in peripheral blood Scr, eGFR and other indexes in the three groups before operation $(\mathrm{P}>0.05)$. Compared with before operation, Scr level at $24 \mathrm{~h}$ and $72 \mathrm{~h}$ after operation in the simple hydration group was significantly increased, while eGFR level was significantly decreased, which is of statistical significance $(\mathrm{P}<0.05)$. Compared with $24 \mathrm{~h}$ after operation, Scr level increased significantly at $72 \mathrm{~h}$ after operation, while eGFR level decreased more significantly, which bears statistical significance $(\mathrm{P}<0.05)$. Scr level at $24 \mathrm{~h}$ and $72 \mathrm{~h}$ after surgery in intensive statin group and the Shenduning prescription group was lower than that in simple hydration group at the same period, while eGFR level at $24 \mathrm{~h}$ and $72 \mathrm{~h}$ after operation was higher than that in simple hydration group at the same period, which is of statistical significance $(\mathrm{P}<0.05)$. The Scr and eGFR levels of the intensive statin group at $24 \mathrm{~h}$ and $72 \mathrm{~h}$ after operation were not significantly different from those of the Shenduning prescription group $(\mathrm{P}>0.05)$. See Table 2 . 
Table 2. Comparison of markers of renal injury in the three groups

\begin{tabular}{|c|c|c|c|c|}
\hline Observation indexes & Time & $\underset{(n=53)}{\text { Simple hydration group }}$ & $\begin{array}{l}\text { Intensive statin group } \\
(n=53)\end{array}$ & $\begin{array}{l}\text { Shenduning prescription group } \\
(n=53)\end{array}$ \\
\hline \multirow[t]{3}{*}{$\operatorname{Scr}(\mu \mathrm{mol} / \mathrm{L})$} & Preoperation & $84.84 \pm 17.26$ & $83.65 \pm 18.34$ & $84.01 \pm 18.85$ \\
\hline & Postoperation $24 \mathrm{~h}$ & $103.16 \pm 15.53$ & $92.35 \pm 14.67^{\wedge}$ & $94.82 \pm 15.06^{\boldsymbol{\Lambda}}$ \\
\hline & Postoperation $72 \mathrm{~h}$ & $117.29 \pm 25.31$ & $95.87 \pm 25.24^{\wedge}$ & $98.26 \pm 23.12^{\Delta}$ \\
\hline \multirow{2}{*}{$\begin{array}{c}\text { eGFR } \\
\left(\mathrm{ml} / \mathrm{min} / 1.73 \mathrm{~m}^{2}\right)\end{array}$} & Postoperation $24 \mathrm{~h}$ & $84.18 \pm 19.74$ & $91.08 \pm 21.57^{\Perp}$ & $90.96 \pm 22.73^{\wedge}$ \\
\hline & Postoperation $72 \mathrm{~h}$ & $77.84 \pm 17.38$ & $86.49 \pm 22.07^{\mathbf{\Lambda}}$ & $85.79 \pm 20.14^{\boldsymbol{\Lambda}}$ \\
\hline
\end{tabular}

Notes: ${ }^{\wedge}$ refers to $\mathrm{P}<0.05$ compared with simple hydration group at the same period

\subsection{Comparison of oxidative stress indexes in the three groups}

There were no significant differences in MDA and SOD levels in the three groups before operation ( $P>0.05)$. MDA levels in intensive statin group and the Shenduning prescription group at $24 \mathrm{~h}$ and $72 \mathrm{~h}$ after surgery were lower than those in simple hydration group at the same period, which is of statistical significance $(\mathrm{P}<0.05)$. The MDA level of Shenduning prescription group at $72 \mathrm{~h}$ after operation was lower than that of intensive statin group at the same period, and the difference was statistically significant $(\mathrm{P}<0.05)$. SOD level at $24 \mathrm{~h}$ and $72 \mathrm{~h}$ after operation in intensive statin group and the Shenduning prescription group was higher than that in simple hydration group at the same period, bearing statistical significance $(\mathrm{P}<0.05)$. SOD level in Shenduning prescription group at $72 \mathrm{~h}$ after operation was higher than that at the same period of intensive statin group, with statistical significance $(\mathrm{P}<0.05)$. See Table 3 .

Table 3. Comparison of oxidative stress indexes in the three groups

\begin{tabular}{|c|c|c|c|c|}
\hline Observation indexes & Times & $\begin{array}{l}\text { Simple hydration group } \\
\qquad(\mathrm{n}=53)\end{array}$ & $\begin{array}{l}\text { Intensive statin group } \\
\qquad(\mathrm{n}=53)\end{array}$ & $\begin{array}{l}\text { Shenduning prescription group } \\
\qquad(\mathrm{n}=53)\end{array}$ \\
\hline \multirow[t]{2}{*}{ MAD (U/L) } & Preoperation & $25.08 \pm 3.04$ & $25.79 \pm 2.79$ & $24.73 \pm 3.17$ \\
\hline & $72 \mathrm{~h}$ postoperation & $39.12 \pm 4.17$ & $31.51 \pm 2.83^{\wedge}$ & $26.77 \pm 2.46^{\Delta \cdot}$ \\
\hline \multirow{2}{*}{$\mathrm{SOD}(\mathrm{mg} / \mathrm{L})$} & $24 \mathrm{~h}$ postoperation & $22.38 \pm 3.09$ & $29.74 \pm 3.63^{\Delta}$ & $28.73 \pm 3.38^{\wedge}$ \\
\hline & $72 \mathrm{~h}$ postoperation & $14.09 \pm 1.83$ & $20.09 \pm 2.24^{\mathbf{\Lambda}}$ & $27.76 \pm 2.76^{\boldsymbol{\Delta}}$ \\
\hline
\end{tabular}

Notes: ${ }^{\wedge}$ refers to $\mathrm{P}<0.05$ compared with simple hydration group at the same period; "refers to $\mathrm{P}<0.05$ compared with intensive statin group at the same period

\subsection{Comparison of CIN incidence in the three groups within $\mathbf{7 2}$ hours after operation}

Taking the absolute value of Scr increased $\geq 0.05 \mathrm{mg} / \mathrm{dl}(44.2 \mu \mathrm{mol} / \mathrm{L})$ or the relative value of Scr increased $\geq 25 \%$ as the diagnostic criteria of $\mathrm{CIN}$ incidence, we found that there were 8 cases $(15.1 \%)$ in simple hydration group, 3 cases $(5.7 \%)$ in intensive statin group, and 2 cases $(3.8 \%)$ in Shenduning prescription group. There was no significant difference between the three groups $\left(x^{2}=5.194, \mathrm{P}=0.075\right)$. The $\mathrm{CIN}$ incidence of simple hydration group was higher than that of intensive statin group, but the difference was not significant $\left(x^{2}=2.536, \mathrm{P}=0.111\right)$. However, the CIN incidence in simple hydration group was higher than that in Shendouning prescription group, and the difference was statistically significant $\left(x^{2}=3.975, \mathrm{P}=0.046\right)$. There was no significant difference between intensive statin group and Shenduning prescription group in CIN incidence $\left(x^{2}=0.210, \mathrm{P}=0.647\right)$.

\subsection{Comparison of the incidence of postoperative renal dialysis and abnormal liver function in the three groups}

No cases were reported to receive renal dialysis treatment in the three groups within 1 week after operation. The incidence of abnormal liver function was as follows: 2 cases (3.8\%) in simple hydration group, 7 cases $(13.2 \%)$ in intensive statin group and 1 case (3.8\%) in the Shenduning prescription group. There was statistical significance in the incidence of abnormal liver function among the three groups $\left(x^{2}=6.616, \mathrm{P}=0.037\right)$. The incidence of abnormal liver function in simple 
hydration group was lower than that in intensive statin group, with insignificant difference $\left(x^{2}=3.306, \mathrm{P}=0.081\right)$, while it was higher in simple hydration group than in Shenduning prescription group, and the difference was not statistically significant $\left(x^{2}=0.343, \mathrm{P}=0.558\right)$. Moreover, the incidence of abnormal liver function in intensive statin group was higher than that in Shenduning prescription group, and the difference was statistically significant $\left(x^{2}=4.867, \mathrm{P}=0.027\right)$.

\section{Discussion}

$\mathrm{CIN}$ is a common and sever complication of patients after coronary interventional treatment. As the patients receiving such treatment are sharply increasing, especially those aging ones, the incidence of CIN is also increasing significantly ${ }^{[1]}$. Therefore, $\mathrm{CIN}$ is attracting more and more attention from cardiovascular therapists. At present, we have no idea of the exact mechanism of CIN pathogenesis, and there still lacks effective prevention and treatment methods in clinic practice. And currently, the most recognized method for the prevention and treatment of CIN is to apply adequate hydration treatment at perioperative period ${ }^{[4-5]}$. Statins have multiple cardiovascular protective effects, such as lipid regulation, anti-inflammation, anti-oxidation, inhibition of myocardial fibrosis and promotion of vascular regeneration. A number of studies ${ }^{[8-10]}$ have shown that large doses of statins may have a certain preventive effect on CIN, and they will reduce the incidence of perioperative CIN when combination treatment of intensive statins with hydration therapy was applied to patients receiving coronary intervention at the perioperative period. However, other studies found that intensive statin therapy may not reduce the incidence of CIN incidence at the perioperative period after coronary intervention ${ }^{[11]}$. Therefore, the treatment of applying intensive statin therapy to patients at the perioperative period to prevent CIN incidence needs to be further confirmed. In addition, drugs such as antioxidants, diuretics and vasodilators may also have a certain effect ${ }^{[12-14]}$. However, although there are numerous clinical methods, we have not found asatisfactory clinical treatment for CIN.

In recent years, traditional Chinese medical doctors have studied a lot on $\mathrm{CIN}$, and they find that integrated prevention and treatment of traditional Chinese and western medicine may be an effective means to prevent and treat CIN. Li et al. ${ }^{[15]}$ found that compound Danshen dropping pills combined with hydration treatment can better prevent the occurrence of CIN in coronary intervention. Zhao et al. ${ }^{[16]}$ observed that internal administration of Xuefu Zhuyu pill decoction combined with hydration therapy during perioperative period of coronary intervention can effectively remove oxygen free radicals in patients and reduce the incidence of CIN. Traditional Chinese medicine believes that the kidney is the main organ to transpire and gasify water, and the contrast agent is the venom from outside, which will inhibits the kidney's transportation and discharge of water in our body. Just as "Simple Question and Comment on Heat Disease" said, "the kidney is water viscus and is in charge of our body fluid", so the transpiration and gasification of all our body fluids depend on the kidney. Moreover, the deficiency of viscera is mainly caused by the internal disorders of our body. The elderly are the most prone population of cardiovascular and cerebrovascular diseases, and their rate of coronary intervention is significantly higher than that of other populations. According to "Simple Question and Comment on Heat Disease", "When evil forces gather together, it definitely indicates deficiency of vital energy in our body". The elderly are relatively weak in their body energy, and are usually unable to fight against evil forces, so they are prone to be attacked by the evil forces. Therefore, the main cause and pathogenesis of this disease is the invasion of external evil forces, which will inhibits the transpiration and gasification of our body fluids and leave some toxic residual in our body. Additionally, the deficiency in our viscera will inhibit the ordinary transportation of our body fluids, leading to obstruction in Qi-Blood circulation. As time passes, it will lead to blood viscidity and stasis and eventually result in reduced glomerular filtration rate, reduced glomerular perfusion, and renal tubular damage and so on. The symptom of this disease appears in kidney, but it is closely related to lung, spleen and the triple energizers. The pathology of this disease shows the deficiency in spleen and kidney, but actually it is caused by "dampness", "toxicity" and "stasis" in our whole body.

Shengduning prescription is made of nine kinds of Chinese medicine, namely, astragalus membranaceus, herba epimedii, radix pseudostellariae, prepared rhubarb, salvia miltiorrhiza, Chinese agarwood powder, peach kernel, prepared sealwort, cassia twig. And it is proved to have significant clinical curative effect on chronic renal failure through a large number of clinical and animal experiments ${ }^{[17-19]}$. Astragalus membranaceus and herba epimedii are the sovereign drugs in this prescription. When the two medicines are used together, it will help to warm Yang and reinforce Qi, tonify our kidney and invigorate our spleen, and nourish our lung and strengthen our body's resistance and eliminate evil. When prepared rhubarb, salvia miltiorrhiza, Chinese agarwood powder and peach kernel are used together, they can remove blood stasis and collaterals. Prepared rhubarb can clear heat and remove toxicity, as well as remove dampness and discharge fouling residuals in our body. As an associated medicine, it can help to alleviate deficiency in our spleen and kidney and remove "dampness", "toxicity" and "stasis" in our whole body. When associated with radix pseudostellariae and prepared sealwort, this prescription can supplement Qi and nourish Yin, as well as nourish our lung, spleen and kidney. These two kinds of 
medicine can alleviate the warmness and dryness of the sovereign drugs. When associate with cassia twig, it can warm and activate meridian, as well as warm Yang and convert Qi, which can help the sovereign drugs to further develop the function of warming Yang and benefit Qi while helping minister drugs to promote blood circulation and remove meridian obstruction. The whole prescription used several medicines together to attack and tonify the deficiency in the spleen and kidney, so as to nourish the deficiency in spleen and kidney while removing dampness toxicity and stasis. Such a method is in line with the pathogenesis of CIN. In this study, traditional Chinese medicine prescriptions combined with hydration therapy were used to prevent the occurrence of CIN after coronary intervention treatment. The Scr level in peripheral blood of patients in Shenduning prescription group at $24 \mathrm{~h}$ and $72 \mathrm{~h}$ afteroperation was significantly lower than that in simple hydration group, while the eGFR level was significantly higher than that in simple hydration group $(\mathrm{P}<0.05)$, but there was no statistical significance compared with that inintensived statin group $(\mathrm{P}>0.05)$. It is suggested that Shenduning prescription combined with hydration therapy has obvious protective effect on renal injury caused by contrast agent, and the effect is similar to that of intensive statin therapy. According to the results of this study, the MDA level of peripheral blood in Shenduning prescription group at $72 \mathrm{~h}$ after operation was significantly lower than that of simple hydration group and intensive statin group, while the SOD level was significantly higher than that of simple hydration group and intensive statin group, which bears statistical significance $(\mathrm{P}<0.05)$. All these indicate that Shenduning prescription can strongly inhibit lipid peroxidation and remove oxygen free radicals, whose effect on anti-oxidative stress is stronger than that of intensive treatment with statins. The incidence of CIN after elective coronary intervention was significantly lower in the Shenduningfang group than that in simple hydration group $(\mathrm{P}<0.05)$, but there was no significant difference with that of intensive statin groups $(\mathrm{P}>0.05)$, suggesting that Shenduning prescription combined with hydration therapy may reduce the incidence of CIN after elective coronary intervention. The efficacy may be comparable to intensive statin therapy at perioperative period. In this study, it was also observed that the number of cases of abnormal liver function in the Shenduning prescription group was significantly less than that in intensive statin group $(\mathrm{P}<0.05)$, and there was no statistical significance with that in simple hydration group $(\mathrm{P}>0.05)$, suggesting that Shendouning prescription had less side effects on liver function. Therefore, we can claim that Shenduning prescription combined with hydration therapy was very safe.

In conclusion, Shenduning prescription combined with hydration therapy may play a role in preventing CIN occurrence after elective coronary intervention by protecting renal function and anti-oxidative stress and other mechanisms, and the efficacy may be equivalent to that of intensive statins combined with hydration therapy, but it owns stronger effect on antioxidative stress and the higher safety in clinic practice.

As a prospective clinical study, this study bears certain innovative and guiding value for future clinic practice, but at the same time, it also has significant limitations. First of all, this study was non-double-blind study, with few cases enrolled. Secondly, the grouping of patients can be further refined, and the cross therapy of Shenduning prescription and intensive statin can be taken into account. Thirdly, the specific action mechanism of Shendouning prescription is unknown, and it is necessary to conduct in-depth studies on its detailed mechanism of cell signaling pathway and target protein in future studies.

\section{References}

[1] Jing Wang, Chunyu Zhang, Zhina Liu, et al. Risk factors of contrast-induced nephropathy after percutaneous coronary intervention: a retrospective analysis[J]. J Int Med Res. 2021; 49(4): 03000605211005972. doi: $10.1177 / 03000605211005972$.

[2] Prit Kusirisin, Siriporn C. Chattipakorn, Nipon Chattipakorn, et al. Contrast-induced nephropathy and oxidative stress: mechanistic insights for better interventional approaches[J]. J Transl Med. 2020; 18: 400. doi: 10.1186/s12967-02002574-8.

[3] Ashkan Heshmatzadeh Behzadi, Behzad Amoozgar, Shalini Jain, et al. Trimetazidine reduces contrast-induced nephropathy in patients with renal insufficiency undergoing coronary angiography and angioplasty[J]. Medicine (Baltimore). 2021; 100(10): e24603. doi: 10.1097/MD.0000000000024603.

[4] Jiantong Hou, Gaoliang Yan, Bo Liu, et al. The Protective Effects of Enalapril Maleate and Folic Acid Tablets against Contrast-Induced Nephropathy in Diabetic Rats[J]. Biomed Res Int. 2018; 2018: 4609750. doi: 10.1155/2018/4609750.

[5] Yanfei Li, Ke Ren. The Mechanism of Contrast-Induced Acute Kidney Injury and Its Association with Diabetes Mellitus[J]. Contrast Media Mol Imaging. 2020; 2020: 3295176. doi: 10.1155/2020/3295176.

[6] Hui-Chao Pan, Xian-Hao Wu, Qian-Li Wan, et al. Analysis of the risk factors for contrast-induced nephropathy in over-aged patients receiving coronary intervention[J]. Exp Biol Med (Maywood). 2018; 243(12): 970-975. doi: $10.1177 / 1535370218799973$.

[7] Li Lei, Yibo He, Zhaodong Guo, et al. A Simple Nomogram to Predict Contrast-Induced Acute Kidney Injury in Pa- 
tients with Congestive Heart Failure Undergoing Coronary Angiography[J]. Cardiol Res Pract. 2021; 2021: 9614953. doi: 10.1155/2021/9614953.

[8] Min Liang, Shicheng Yang, Naikuan Fu. Efficacy of short-term moderate or high-dose rosuvastatin in preventing contrast-induced nephropathy[J]. Medicine (Baltimore). 2017; 96(27): e7384. doi: 10.1097/MD.0000000000007384.

[9] Arezoo Khosravi, Mitra Dolatkhah, Hesam Sadat Hashemi, et al. Preventive Effect of Atorvastatin (80 mg) on Contrast-Induced Nephropathy After Angiography in High-Risk Patients: Double-Blind Randomized Clinical Trial[J]. Nephrourol Mon. 2016; 8(3): e29574. doi: 10.5812/numonthly.29574.

[10] Hamid Sanei, Alireza Hajian-Nejad, Amirreza Sajjadieh-Kajouei, et al. Short term high dose atorvastatin for the prevention of contrast-induced nephropathy in patients undergoing computed tomography angiography[J]. ARYA Atheroscler. 2014; 10(5): 252-258.

[11] Wei-Jie Bei, Shi-Qun Chen, Hua-Long Li, et al. Comparing common doses (double-dose vs usual-dose) of atorvastatin for preventing contrast-induced acute kidney injury and mortality after coronary angiography[J]. Medicine (Baltimore). 2017; 96(30): e7501. doi: 10.1097/MD.0000000000007501.

[12] Laura Vicente-Vicente, David González-Calle, Alfredo Ginés Casanova, et al. Quercetin, a Promising Clinical Candidate for The Prevention of Contrast-Induced Nephropathy[J]. Int J Mol Sci. 2019; 20(19): 4961. doi: 10.3390/ijms20194961.

[13] Dongling Liu, Feng Gao, Li Li, et al. The prophylactic effect of alprostadil on contrast-induced nephropathy in renal insufficiency patients after percutaneous coronary intervention[J]. Am J Transl Res. 2021; 13(4): 3766-3772.

[14] Yi-Hsin Chen, Yun-Ching Fu, Ming-Ju Wu. Does Resveratrol Play a Role in Decreasing the Inflammation Associated with Contrast Induced Nephropathy in Rat Model?[J]. J Clin Med. 2019; 8(2): 147. doi: 10.3390/jcm8020147.

[15] Cailan Li, Qian Li, Jiamin Xu, et al. The Efficacy and Safety of Compound Danshen Dripping Pill Combined with Percutaneous Coronary Intervention for Coronary Heart Disease[J]. Evid Based Complement Alternat Med. 2020; 2020: 5067137. doi: 10.1155/2020/5067137.

[16] Jingjing Zhao, Huahua Liu, Buyun Xu, et al. The Role of Xuefu Zhuyu Decoction in Prevention of Contrast-Induced Nephropathy after Percutaneous Coronary Intervention[J]. Evid Based Complement Alternat Med. 2020; 2020: 5419016. doi: 10.1155/2020/5419016.

[17] QIAN Lu, FU Xiaojun, HE Liqun. Effects of Shendu Ning Agent on the release of Vasoactive Substances in Patients with Chronic Kidney Disease[J]. Zhejiang Journal of Integrated Traditional Chinese and Western Medicine. 2011; 21(4): 219-224.

[18] Xiong Rongbing, Zhang Ting, He Liqun, et al. Experimental Study on the Protective Effect of Shenduning Granule on Renal Failure Model Rats and its Mechanism[J]. Journal of Emergency in Traditional Chinese Medicine. 2017; 26(5): 775-778. doi:10.3969/j.issn.1004-745X.2017.05.007.

[19] Xiong Rongbing, He Liqun, FU Xiaojun. Experimental Study on the Effects of Shenduning Particles on Antioxidant and MCP-1 Inflammatory Factors in Renal Tissues of Chronic Renal Failure[J]. Chinese Journal of Integrated Traditional and Western Nephrology. 2018; 19(4): 288-291. 